\title{
EVALUACIÓN DEL PROCESO DE COMPOSTAJE DE RESIDUOS AVÍCOLAS EMPLEANDO DIFERENTES MEZCLAS DE SUSTRATOS
}

Assessment of the composting process of poultry manure using different mixtures of substrates

\section{María del Mar DELGADO ARROYO ${ }^{1 *}$, Karla Luz MENDOZA LÓPEZ², María Isabel GONZÁLEZ1, José Luis TADEO LLUCH y José Valero MARTÍN SÁNCHEZ ${ }^{1}$}

${ }^{1}$ Departamento de Medio Ambiente, Instituto Nacional de Investigación y Tecnología Agraria y Alimentaria (INIA), km. 7.5 carretera de La Coruña, 28040 Madrid, España

${ }^{2}$ Universidad Autónoma de Madrid, Campus Cantoblanco, km 15 carretera Colmenar, 28049 Madrid, España

*Autora para correspondencia: delgado@inia.es

(Recibido: febrero 2018; aceptado: noviembre 2018)

Palabras clave: gallinaza, paja, huevos y ceniza, pila semiindustrial, caracterización física y química

\section{RESUMEN}

Los residuos agropecuarios, en particular los procedentes de la actividad avícola, pueden contribuir a la contaminación del suelo, agua y aire, y generar un impacto negativo, siendo el proceso de compostaje una alternativa. Este trabajo ha tenido como objetivo reutilizar estos residuos y evaluar el proceso de compostaje a nivel de laboratorio en cuatro tratamientos: gallinaza + paja $(\mathrm{G}+\mathrm{P})$, gallinaza + paja + cenizas $(\mathrm{G}+\mathrm{P}+\mathrm{C})$, gallinaza + paja + huevos $(\mathrm{G}+\mathrm{P}+\mathrm{H})$, y gallinaza + paja + huevos + cenizas $(\mathrm{G}+\mathrm{P}$ $+\mathrm{H}+\mathrm{C}$ ), todos ellos con la cantidades adecuadas para que la relación $\mathrm{C} / \mathrm{N}<20$ sea idónea para el compostaje. Adicionalmente, para estudios comparativos se realizó una pila a escala semiindustrial de $20 \mathrm{~m}^{3}$ en condiciones ambientales y un bioensayo del compost final para medir su fitoxicidad. Con el fin de evaluar el proceso de compostaje, en los tratamientos y en la pila se realizaron análisis físicos y químicos de los siguientes parámetros: $\mathrm{pH}$, conductividad eléctrica $(\mathrm{CE})$, materia orgánica $(\mathrm{MO})$, nitrógeno $(\mathrm{N})$ y relación carbono:nitrógeno $(\mathrm{C} / \mathrm{N})$, con sus respectivos análisis de varianza de múltiple rango. Para determinar el efecto de los tratamientos y comparar las medias se utilizó la prueba de la mínima diferencia significativa a un nivel de significación de $\mathrm{p} \leq 0.05$. Los resultados obtenidos a partir de las diferentes mezclas de sustratos en el tiempo en que se realizó el compostaje, reflejan una buena calidad agronómica, ya que éste no presentó concentraciones de compuestos fitotóxicos que afecten los cultivos y por lo tanto puede utilizarse como abono orgánico y sustrato de cultivos.

Key words: poultry manure, straw, eggs and ash, semi-industrial pile, physical and chemical characterization

\begin{abstract}
Agricultural residues, particularly those from poultry activity, may have a negative impact, contributing to soil, water and air pollution. The composting process is an alternative to this. The objective of this work is to reuse these residues and evaluate the composting process at the laboratory level in four treatments: poultry manure + straw $(\mathrm{P}+\mathrm{S})$, poultry manure + straw + ash $(\mathrm{P}+\mathrm{S}+\mathrm{A})$, poultry manure + straw + eggs $(\mathrm{P}+\mathrm{S}+\mathrm{E})$, and poultry manure + straw + eggs + ash $(\mathrm{P}+\mathrm{S}+\mathrm{E}+\mathrm{A})$, all of them in
\end{abstract}


the adequate proportions so that the carbon:nitrogen ratio is suitable for composting $(\mathrm{C} / \mathrm{N}<20)$. Additionally, for comparative studies, a semi-industrial pile level of 20 $\mathrm{m}^{3}$ under environmental conditions and a bioassay of the final compost were carried out to measure its phytotoxicity. In order to evaluate the composting process, the physical and chemical parameters $(\mathrm{pH}$, electrical conductivity [EC], organic matter $[\mathrm{OM}]$, nitrogen $[\mathrm{N}]$ and $\mathrm{C} / \mathrm{N}$ ratio) were analyzed, with their respective analysis of variance of multiple range, to determine the effect of the treatments; to compare the means, the least significant difference test was used at a level of significance of $p \leq$ 0.05 . The results obtained from the different mixes of substrates during the time the compost was done, showed a good agronomic quality, not presenting concentrations of phytotoxic compounds that affect crops; therefore, it can be used as organic fertilizer and cultivation substrate.

\section{INTRODUCCIÓN}

En los últimos años una de las actividades económicas que ha tenido mayor crecimiento en España es la actividad avícola, debido a los mejores niveles de eficiencia productiva y rendimiento tecnológico. Esta actividad representa el $13.4 \%$ de producción a nivel nacional (MAGRAMA 2014). Constituye un sector importante dentro de la producción de alimentos básicos, el cual ha generado un incremento de residuos avícolas (excretas).

En España, la actividad avícola genera una gran cantidad de residuos orgánicos que pueden causar problemas de polución debido a la gran cantidad de nitrógeno, fósforo y azufre que contienen y que ocasionan eutrofización de las aguas (Smith et al. 2001). A pesar de estos efectos negativos, dichos residuos pueden ser reutilizados, ya que contienen compuestos orgánicos e inorgánicos que son fuente de nutrientes (nitrógeno, fósforo y potasio) y microorganismos beneficiosos para la agricultura si se les da un tratamiento adecuado (Hernández y Rodríguez 2013). De este modo, se considera importante la implementación de métodos de tratamiento más sostenibles que sean compatibles con el medio ambiente, impulsando técnicas y sistemas de producción como el compostaje que ayuden a mitigar impactos ambientales (Arrigo et al. 2005, Celaya y Castellanos 2011).

El compostaje es un método que permite la transformación de la materia orgánica (MO) en un producto con precursores de sustancias húmicas a través de la descomposición aeróbica, que da como resultado un producto final estable (Soto 2003, Riera et al. 2014). Para el compostaje, los residuos deben mezclarse en proporciones tales que la relación carbono:nitrógeno $(\mathrm{C} / \mathrm{N})$, la humedad (40-60 \%) y la aireación sean adecuadas para la actividad microbiana, con el fin de que ésta permita la modificación de la estructura de los materiales para que los nutrientes estén disponibles (Hernández et al. 2013).

Durante este proceso de biodegradación, la actividad metabólica de los microorganismos mesófilos y termófilos genera una actividad biológica que permite la descomposición de la MO y su mineralización (Sztern y Pravia 2008). También hay que tener en cuenta las condiciones óptimas de temperatura (50-60 ${ }^{\circ} \mathrm{C}$ ), humedad y oxigenación necesarias para obtener un producto de calidad (Torrentó 2011).

La principal característica del compost es su capacidad de aplicación y utilización en todo tipo de suelos, incluyendo zonas áridas y semiáridas, así como ecosistemas afectados por incendios y sequías (Guenon y Gros 2015). El compost es especialmente rico en proteínas y minerales. Proporciona nutrientes y restaura las propiedades físicas y químicas de los suelos que han sido alteradas por las actividades agrícolas (Nieto 2002). También ayuda a disminuir el uso de agroquímicos, disminuyendo así la toxicidad y evitando la erosión y degradación de los suelos (Ramírez et al. 2014).

La utilización de abono orgánico tiene como ventaja la conservación, restauración y mejoramiento de las características de los suelos, así como el equilibrio biológico, físico, químico y ecológico de los mismos, además del restablecimiento de la flora microbiana. Esto se debe a que aporta principalmente nitrógeno, fósforo, potasio y MO, lo que permite mantener el nivel óptimo de los suelos y preservar los ecosistemas (Morisaki et al. 1989, Matheus et al. 2007).

La aplicación de compost inmaduro e inestable puede afectar la inmovilización de nitrógeno en el suelo, restringir el crecimiento de la planta y causar efectos fitotóxicos (Delgado et al. 2007, 2010). Para evitar lo anterior, se debe evaluar la calidad del compost que será utilizado como enmiendas y como abono para mejorar la productividad y disminuir el impacto ambiental. Es importante aprovechar este recurso rico 
en proteínas y minerales basado en las propiedades de sus parámetros físicos y químicos (Liao et al. 2013).

Actualmente, este abono se aplica en sistemas agroecológicos, ya que tiene propiedades como mejorador de suelos, capacidad de retención del agua y permeabilidad, lo cual mejora la estructura del suelo y proporciona los nutrientes necesarios para el crecimiento de los cultivos. Además, es un producto libre de fertilizantes sintéticos y bajo costo (Fang y Wong 1999, Magdi et al. 2004, Roca et al. 2008). Tautges et al. (2018) encontraron una gran productividad en un cultivo de trigo, cuando su fertilización nitrogenada procedía de un abono orgánico de gallinaza.

Se ha llevado a cabo una investigación cuyo objetivo es estudiar un proceso de compostaje con gallinaza, residuos de huevos y cenizas a nivel de laboratorio y a escala semiindustrial, para reutilizar los residuos avícolas y producir compost que cumpla con los parámetros de calidad necesarios para considerase como abono orgánico.

\section{MATERIALES Y MÉTODOS}

El ensayo se desarrolló en el Instituto Nacional de Investigación y Tecnología Agraria y Alimentaria (INIA) situado en Madrid, España.

Se realizó un estudio a pequeña escala de laboratorio y otro a escala semiindustrial para comprobar la evolución de los parámetros carbono oxidable, $\mathrm{CE}, \mathrm{pH}$, nitrógeno y relación $\mathrm{C} / \mathrm{N}$ para identificar la calidad agronómica de este compost, potenciando la técnica y los beneficios de este proceso y garantizando que cumpla con los parámetros del Real Decreto (RD) 506/2013 (BOE 2013) sobre compuestos orgánicos usados como abonos o fertilizantes.

También se midieron la temperatura y la humedad, que son factores necesarios para que el compostaje se realice correctamente, de manera semanal en el ensayo a pequeña escala y de forma quincenal en el ensayo a escala semiindustrial. La temperatura fue medida utilizando sondas de temperatura colocadas en el contenedor y en la pila de compostaje de la marca Hangzhou Kehang Thermometer de sonda corta $(30 \mathrm{~cm})$ y sonda larga (1 m), respectivamente. La humedad del compost se mantuvo en 40 al $60 \%$ mediante riegos constantes y se calculó por el método gravimétrico (MAPA 1994).

\section{Ensayo a pequeña escala}

En el ensayo a pequeña escala se trabajó con residuos avícolas (gallinaza, huevos y cenizas) y paja. La gallinaza fresca, los huevos y las cenizas procedían de una granja de gallinas ponedoras situada en la provincia de Cuenca (España). Los huevos son los restos de huevos rotos con cáscara y las cenizas se recogieron de una incineradora ubicada dentro de la granja, para eliminar restos de animales. La paja procedía de un cultivo de cebada proporcionada por un agricultor de Castilla y León.

El proceso se realizó en un invernadero en contenedores de plástico abiertos de $55 \mathrm{~cm}$ de largo, $35 \mathrm{~cm}$ de ancho y $19 \mathrm{~cm}$ de alto en lo que se incorporaron las combinaciones de gallinaza, paja cortada (5 a 10 $\mathrm{cm})$, huevos y cenizas. Esta mezcla se humedeció con agua corriente del grifo para mantener una humedad constante de 40 a $60 \%$. También se tuvo en cuenta la temperatura $\left(25^{\circ} \mathrm{C}\right)$ y se realizaron volteos semanales según las recomendaciones de Torrentó (2011) y Hernández y Rodríguez (2013). El estudio se llevó a cabo en 12 contenedores, que corresponden a cuatro tratamientos repetidos tres veces (Cuadro I).

El diseño de los tratamientos se realizó teniendo en cuenta la relación $\mathrm{C} / \mathrm{N}<20$ resultante de la mezcla de los diferentes sustratos idónea para que el proceso de compostaje se lleve a cabo.

Cada tratamiento está conformado por:

- Tratamiento 1 (testigo): gallinaza $(7 \mathrm{~kg})+$ paja (200 g).

- Tratamiento 2: gallinaza $(7 \mathrm{~kg})+$ paja $(200 \mathrm{~g})+$ cenizas $(300 \mathrm{~g})$.

- Tratamiento 3: gallinaza $(7 \mathrm{~kg})+$ paja $(200 \mathrm{~g})+$ huevos (864 g).

- Tratamiento 4: gallinaza (7 kg) + paja $(200 \mathrm{~g})+$ huevos (864 g) + cenizas (300 g).

\section{Ensayo a escala semiindustrial}

Para proceder al compostaje a escala semiindustrial se construyó una pila en hilera de $20 \mathrm{~m}^{3}(10 \mathrm{~m}$ de largo, $2 \mathrm{~m}$ de ancho y $1 \mathrm{~m}$ de alto) en la que se incorporaron un total de $8000 \mathrm{~kg}$ de gallinaza y 2000 $\mathrm{kg}$ de paja en nueve capas, alternando gallinaza y paja y empezando y terminado con gallinaza (Cuadro I). La aireación se proporcionó mediante volteos semanales y la humedad se mantuvo entre 40 y $60 \%$ mediante riegos por aspersión.

Los dos ensayos intentaron reflejar cómo se desarrolla un proceso de co-compostaje a pequeña escala (con diferentes sustratos procedentes de un granja de gallinas) y un proceso de compostaje a escala semiindustrial (compostaje real de estiércol de gallinaza). Estos dos procesos de compostaje tuvieron una duración aproximada de cuatro meses hasta alcanzar la etapa de maduración, en la cual se obtuvo el producto. Los muestreos se hicieron semanalmente en tres sectores (superior, medio e 
CUADRO I. DISEÑO EXPERIMENTAL DE LOS RESIDUOS AVÍCOLAS EN CONTENEDOR Y EN PILA

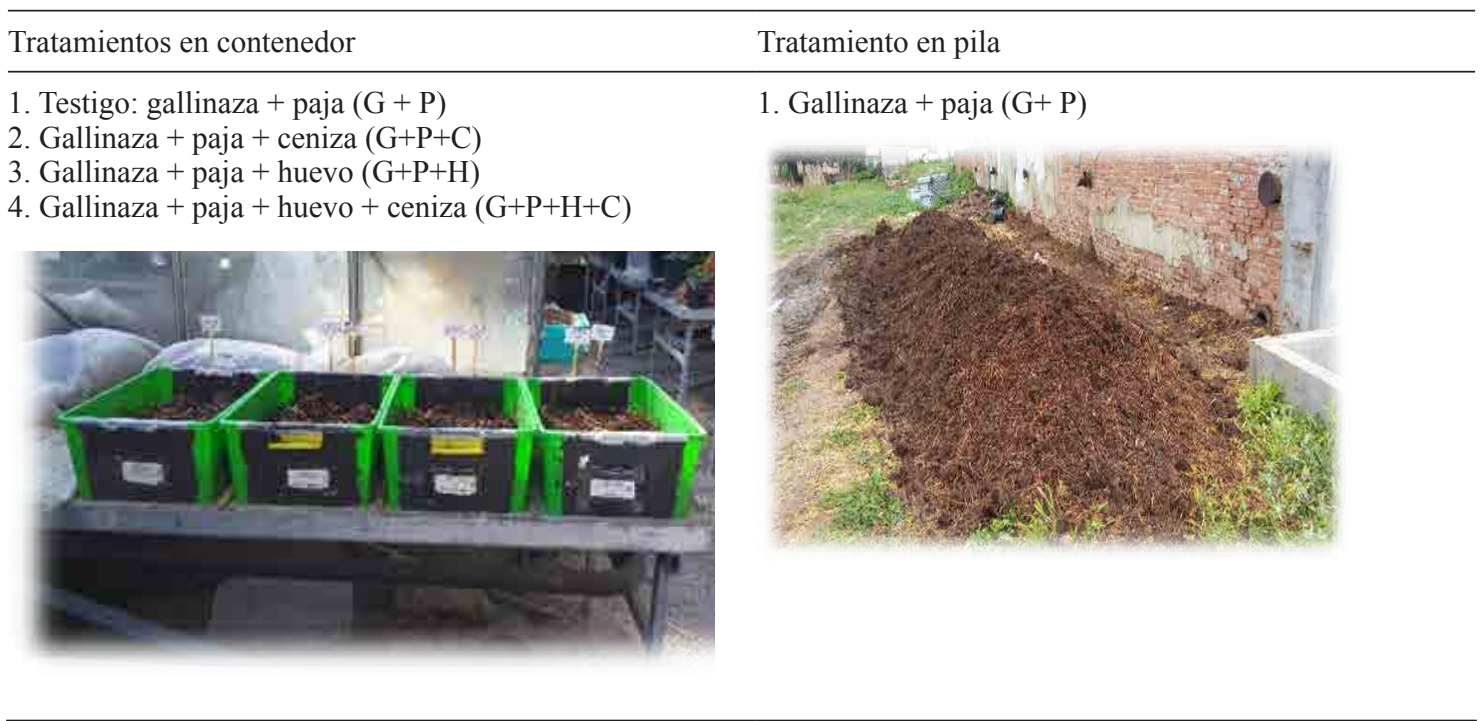

inferior) de los contenedores y de la pila. En cada sector se obtuvieron tres muestreos (uno central y dos laterales) y se extrajo un total de $200 \mathrm{~g}$, el cual se homogeneizó posteriormente hasta obtener una muestra representativa. Las muestras se dispusieron en bolsas de plástico herméticas y se preservaron a $4{ }^{\circ} \mathrm{C}$ para análisis en laboratorio.

\section{Análisis físico y químico del compost del experi- mento a escalas pequeña y semiindustrial}

Los ensayos efectuados del compost Después de secarlo al aire, molerlo en un molino de aspas y tamizarlo en un tamiz con luz de malla de $2 \mathrm{~mm}$, se realizaron los siguientes ensayos al compost del experimento:

- El pH y la conductividad eléctrica $(\mathrm{dS} / \mathrm{m})$, se midieron en una suspensión compost/agua (1:5) utilizando un $\mathrm{pH}$-metro y un conductímetro, respectivamente (MAPA 1994).

- El carbono orgánico oxidable se determinó por el método de Walkey-Black, consistente en la oxidación de la muestra con dicromato potásico, utilizando un factor de oxidación de 1.72 , pues se supone que sólo el $58 \%$ del carbono orgánico presente en la muestra es oxidado por este método (APHA-AWWA-WPCF 2005).

- La concentración de nitrógeno total se determinó siguiendo el método Kjeldahl, que consiste en la digestión de la muestra con ácido sulfúrico concentrado.

- El nitrógeno orgánico, que se mineraliza, pasando a forma amoniacal, se valora por colorimetría junto con el amonio ya existente en la muestra
(Hesse 1971).

- La relación $\mathrm{C} / \mathrm{N}$ se calculó mediante la división de los datos obtenidos de carbono orgánico y nitrógeno total.

- La temperatura se midió mediante una sonda colocada en el contenedor y en la pila de compostaje. La humedad se calculó por el método gravimétrico (MAPA 1994).

Los datos se recolectaron cada semana para garantizar que la humedad del proceso de compostaje se encontrara dentro del intervalo optimo del 40 al $60 \%$.

\section{Análisis de la fitotoxicidad del compost del expe- rimento a escalas pequeña y semiindustrial}

Con base en la metodología empleada por Zucconi y Bertoldi (1981) se realizó un bioensayo de fitotoxicidad con berro (Lepidium sativum) en una cámara de germinación a $25^{\circ} \mathrm{C}$ durante $48 \mathrm{~h}$. Cuando esta prueba muestra un índice de germinación mayor de $50 \%$ indica la ausencia de fitotoxicidad. También se realizó un análisis microbiológico de Salmonella mediante detección, confirmación y recuento de colonias para Escherichia coli, con el fin de comprobar que el compost cumpliera con el RD 506/2013 (BOE 2013) de los compuestos orgánicos usados como abonos o fertilizantes según Escudero de Fonseca y Arias (2012).

\section{Análisis estadístico}

Se realizó un análisis de varianza (ANDEVA) de múltiple rango en los diversos tipos de ensayos 
para determinar el efecto de los tratamientos. Para comparar las medias se utilizó la prueba de diferencia mínima significativa (LSD, por sus siglas en inglés) a un nivel de significancia de $\mathrm{p} \leq 0.05$, utilizando el programa estadístico Statgraphics (SAS/STAT 2004).

\section{RESULTADOS Y DISCUSIÓN}

\section{Temperatura}

La temperatura es un factor importante que varía en función de la actividad biológica de los microorganismos. Presenta cuatro fases: mesófila I, termófila, mesófila II y maduración, y constituye una de las condiciones ambientales determinantes durante el proceso de compostaje (Alexander 1977). En la figura 1 se muestra el perfil de temperatura obtenido en la evolución del compostaje para cada uno de los tratamientos realizados. Se pudo observar que durante el proceso de compostaje el aumento de temperatura fue lento hasta el día 14 (fase mesófila), alcanzando la fase termófila para todo los tratamientos (incluyendo el control el día 21); esto indica que el compost cumple los requisitos de higienización de acuerdo con Mathur (1991), con la eliminación de los microorganismos patógenos.

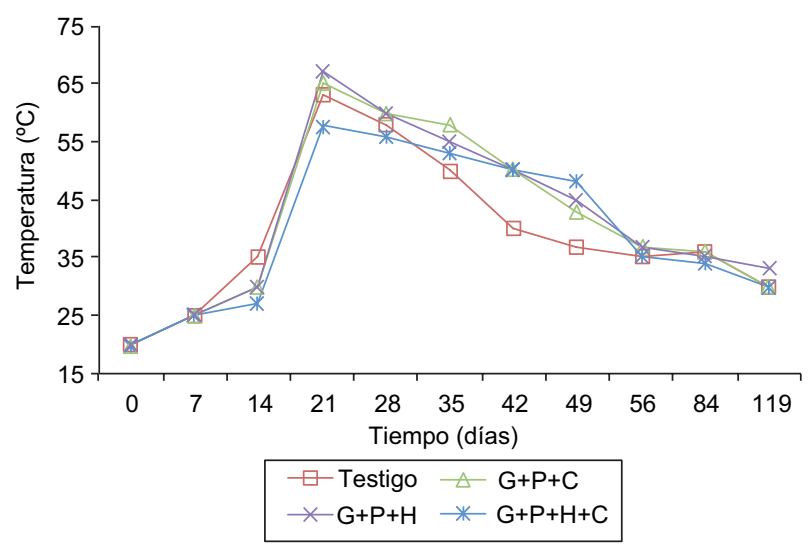

Fig. 1. Variación de la temperatura con el tiempo de los distintos tratamientos testigo en el ensayo a pequeña escala: gallinaza + paja $(\mathrm{G}+\mathrm{P}) ; \mathrm{G}+\mathrm{P}+\mathrm{C}$ : gallinaza + paja + ceniza; $\mathrm{G}+\mathrm{P}+\mathrm{H}$ : gallinaza + paja + huevo; $\mathrm{G}+\mathrm{P}+\mathrm{H}+$ C: gallinaza + paja + huevo + ceniza

Paralelamente se diseñó una pila de compostaje a escala semiindustrial en condiciones reales (Fig. 2), en la cual se alcanzaron temperaturas termófilas por arriba de $60{ }^{\circ} \mathrm{C}$ (como se desea en un proceso de compostaje), ya que éstas se relacionan con el au- mento en la tasa de descomposición de la materia orgánica con gran actividad microbiana según estudios de Pino et al. (2005). La temperatura inicialmente pasó por una etapa termófila, presentando niveles altos $\left(65^{\circ} \mathrm{C}\right)$ debido a la aireación, la cual aumenta la actividad aeróbica microbiana, produciendo una reacción exotérmica (Haug 1993). En la semana 10 las temperaturas en los diferentes tratamientos se estabilizaron sin diferencias entre tratamientos, descendiendo hasta la temperatura ambiente, lo que indica baja actividad microbiana y la posible finalización del compost (Castaldi et al. 2005). Se considera finalizado el proceso cuando la pila llega a la fase de maduración y presenta una temperatura similar a la del ambiente, distinta de la inicial debido a que el final del compostaje ocurrió en época estival, con mayor temperatura que la inicial en época más fría.

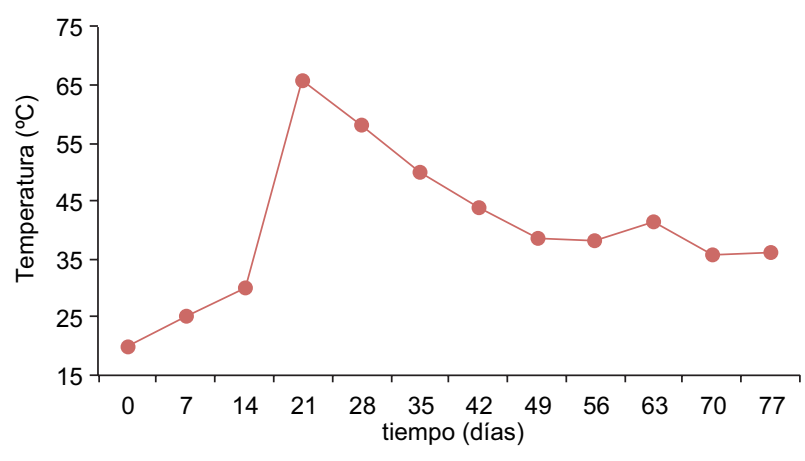

Fig. 2. Variación de la temperatura con el tiempo del tratamiento gallinaza + paja en la pila

\section{Humedad}

Para un buen compostaje los microorganismos presentes necesitan humedad (agua) con el fin de disolver y transportar los nutrientes (Mathur 1991). En este estudio, la humedad en el testigo $\mathrm{G}+$ P presentó el menor porcentaje $(37.37 \pm 8.51 \%)$, seguido por el tratamiento $\mathrm{G}+\mathrm{P}+\mathrm{H}+\mathrm{C}(41.14 \pm 9.45 \%)$, el tratamiento $\mathrm{G}+\mathrm{P}+\mathrm{C}(51.37 \pm 11.70 \%)$ y por último el tratamiento $\mathrm{G}+\mathrm{P}+\mathrm{H}(54.25 \pm 11.87 \%)$, como puede apreciarse en la figura 3. Esto puede atribuirse a los diferentes sustratos de cada tratamiento y a la cantidad de agua utilizada en los riegos. La humedad es un parámetro fundamental en todas las fases del compostaje para que éste se realice adecuadamente: si la humedad al inicio del proceso está por debajo del $35-40 \%$, la actividad microbiana disminuye, pudiendo llegar hasta la inhibición y afectar todo el proceso. 
Por otra parte, una humedad excesiva (mayor a $65 \%$ ) provoca la supresión de la proliferación microbiana debido a la disminución del intercambio gaseoso; esto produce menor disponibilidad de oxígeno y genera un ambiente anaeróbico con malos olores, disminuyendo la velocidad del proceso (Alexander 1977).

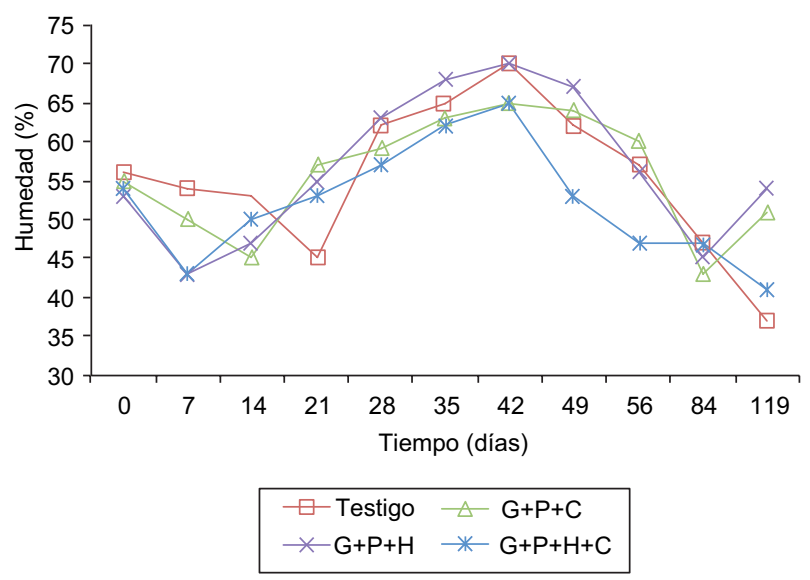

Fig. 3. Variación de la humedad con el tiempo de los distintos tratamientos testigo en el ensayo a pequeña escala: $\mathrm{G}+$ $\mathrm{P}$ : gallinaza + paja; $\mathrm{G}+\mathrm{P}+\mathrm{C}$ : gallinaza + paja + ceniza; $\mathrm{G}+\mathrm{P}+\mathrm{H}$ : gallinaza + paja + huevo; $\mathrm{G}+\mathrm{P}+\mathrm{H}+\mathrm{C}$ : gallinaza + paja + huevo + ceniza

En el caso de la pila, la humedad inicial fue de $55.05 \pm 12.00 \%$ y en la fase final de maduración presentó $36.20 \pm 7.80 \%$ de humedad (Fig. 4). En esta última fase se genera menos calor y la actividad biológica es reducida, por lo que los requerimientos de humedad son menores que en la etapa de descomposición y esto ayuda a obtener un producto estable.

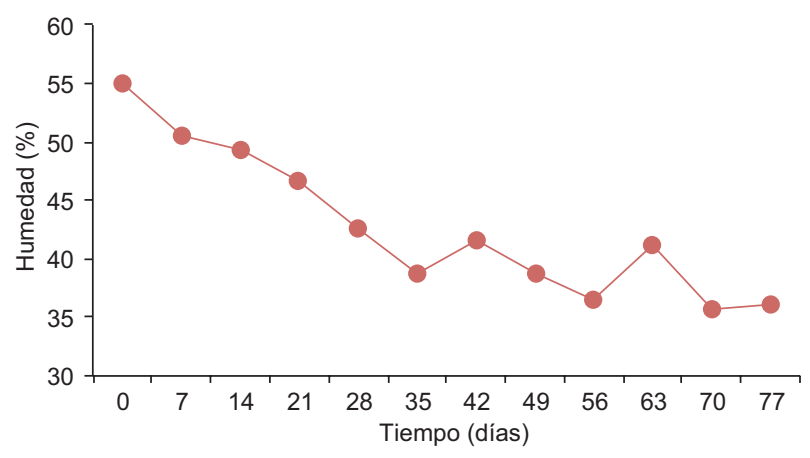

Fig. 4. Variación de la humedad con el tiempo del tratamiento gallinaza + paja en la pila
pH

Los resultados de $\mathrm{pH}$ para el tratamiento $\mathrm{G}+\mathrm{P}$ $+\mathrm{H}$ mostraron un descenso de $8.8 \pm 0.12$ a $8.34 \pm$ 0.12 , lo cual indica actividad microbiana entre la primera y séptima semana. Los demás tratamientos estuvieron por debajo de $8.5 \pm 0.12$ a partir de la octava semana (Fig. 5), lo que indica disminución de la actividad microbiana y por ende estabilización o madurez del compost.

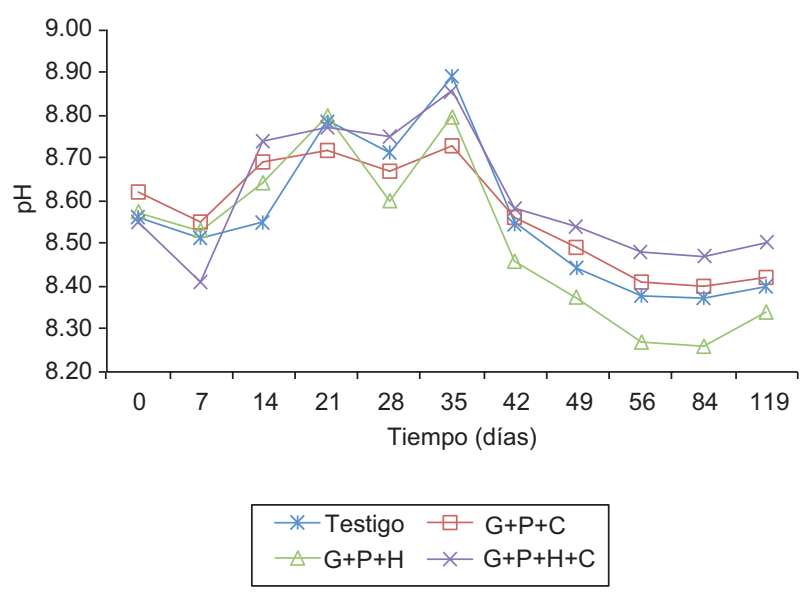

Fig. 5. Variación del pH con el tiempo de los distintos tratamientos testigo en el ensayo a pequeña escala: gallinaza + paja $(\mathrm{G}+\mathrm{P}) ; \mathrm{G}+\mathrm{P}+\mathrm{C}$ : gallinaza + paja + ceniza; $\mathrm{G}+\mathrm{P}+$ $\mathrm{H}$ : gallinaza + paja + huevo; $\mathrm{G}+\mathrm{P}+\mathrm{H}+\mathrm{C}$ : gallinaza + paja + huevo + ceniza

Se realizó un ANDEVA de múltiple rango para determinar el efecto de los tratamientos, y para comparar las medias se utilizó la prueba LSD a un nivel de significación de $\mathrm{p} \leq 0.05$ (Cuadro II). Los

CUADRO II. PRUEBA DE DIFERENCIA MÍNIMA SIGNIFICATIVA(LSD, POR SUS SIGLAS EN INGLÉS) PARA EL $\mathrm{pH}$ POR TRATAMIENTO (MÉTODO $95.0 \%$ LSD)

\begin{tabular}{lccc}
\hline Tratamientos & Repeticiones & Medias & $\begin{array}{c}\text { Grupos } \\
\text { homogéneos }\end{array}$ \\
\hline Testigo & 3 & 8.40 & $\mathrm{~B}$ \\
$\mathrm{G}+\mathrm{P}+\mathrm{C}$ & 3 & 8.42 & $\mathrm{~B}$ \\
$\mathrm{G}+\mathrm{P}+\mathrm{H}$ & 3 & 8.34 & $\mathrm{~A}$ \\
$\mathrm{G}+\mathrm{P}+\mathrm{H}+\mathrm{C}$ & 3 & 8.50 & $\mathrm{C}$ \\
\hline
\end{tabular}

Valores seguidos de la misma letra en la misma columna no difieren significativamente según la prueba LSD $(\mathrm{p}<0.05)$. Testigo: gallinaza + paja $(\mathrm{G}+\mathrm{P}) ; \mathrm{G}+\mathrm{P}+\mathrm{C}$ : gallinaza + paja + ceniza; $\mathrm{G}+\mathrm{P}+\mathrm{H}$ : gallinaza + paja + huevo; $\mathrm{G}+\mathrm{P}+\mathrm{H}+\mathrm{C}$ : gallinaza + paja + huevo + ceniza 
únicos tratamientos que no presentan diferencias significativas son el testigo $(\mathrm{G}+\mathrm{P})$ con el tratamiento $\mathrm{G}+\mathrm{P}+\mathrm{C}$.

Según la figura 6, la evolución del pH en el compostaje dentro de la pila presenta cuatro fases. En la primera fase (mesófila) se observa una disminución del $\mathrm{pH}(8.56 \pm 0.12)$ debido a la acción de los microorganismos sobre la materia orgánica, producto de la liberación de ácidos orgánicos. En esta fase se presenta un descenso del $\mathrm{pH}$ por la presencia de condiciones anaeróbicas, que forman gran cantidad de ácidos orgánicos. En la segunda fase se produce un aumento del $\mathrm{pH}(8.77 \pm 0.12)$ debido a la alcalinización del medio; esto se debe, a su vez, a la pérdida de ácidos orgánicos y la generación de amoníaco por la descomposición de proteínas, de acuerdo con Guerra et al. (2001). Y en la tercera fase el pH $(8.46 \pm 0.12)$ tiende a bajar hasta la fase de maduración (cuarta fase) debido a la formación de compuestos húmicos de acuerdo con Sánchez-Monedero et al. (2001).

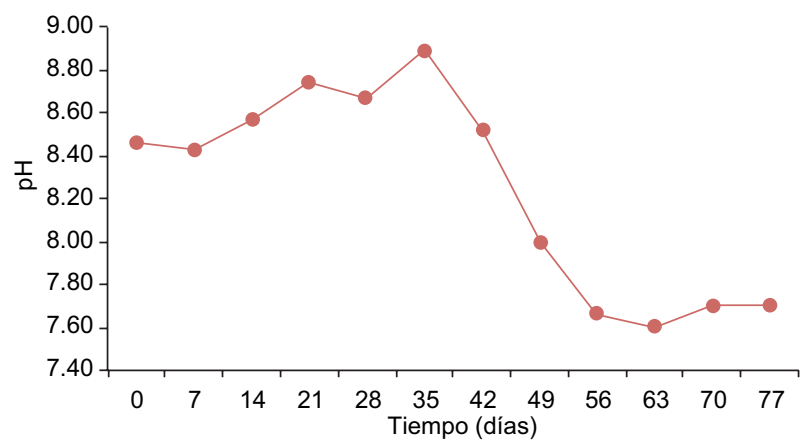

Fig. 6. Variación del pH con el tiempo del tratamiento gallinaza + paja en la pila

\section{Conductividad eléctrica (CE)}

La CE está determinada por la naturaleza y composición del material, principalmente por su concentración de sales y en menor grado por la presencia de iones de amonio o nitrato formados durante el proceso (Sánchez-Monedero et al. 2001). $\mathrm{La} C E$ fue similar al inicio del proceso en los distintos tratamientos, variando de $12.41 \pm 2.70$ a $14.18 \pm$ $3.08 \mathrm{dS} / \mathrm{m}$ (Fig. 7). La evolución de este parámetro presentó aumentos y disminuciones en sus valores, observándose una estabilidad a partir del día 35 y un ligero descenso al final del proceso. Los valores de conductividad obtenidos al final del compostaje (de $3.39 \pm 0.73$ a $5.23 \pm 1.14 \mathrm{dS} / \mathrm{m}$ ) se encuentran dentro de los parámetros que deben cumplir los compuestos orgánicos usados como abonos o fertilizantes (RD 506/2013) (BOE 2013).

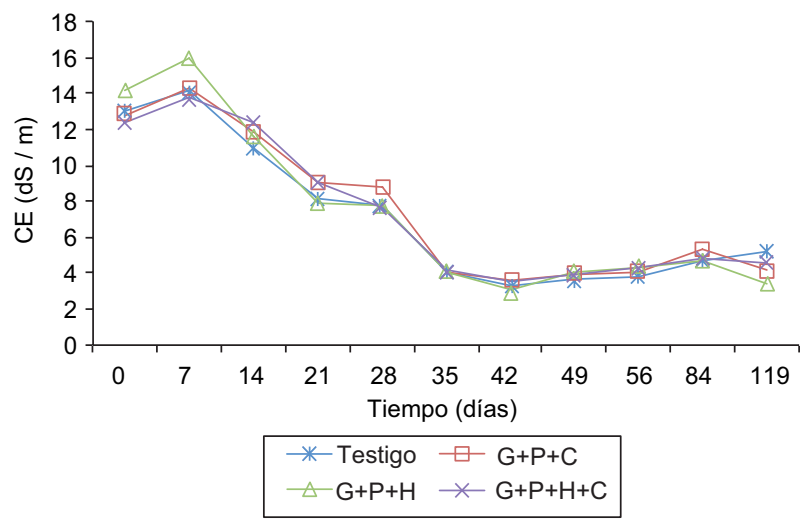

Fig. 7. Variación de la conductividad eléctrica (CE) con el tiempo de los distintos tratamientos testigo en el ensayo a pequeña escala: gallinaza + paja $(\mathrm{G}+\mathrm{P}) ; \mathrm{G}+\mathrm{P}+\mathrm{C}$ : gallinaza + paja + ceniza $; \mathrm{G}+\mathrm{P}+\mathrm{H}$ : gallinaza + paja + huevo; $\mathrm{G}+\mathrm{P}+\mathrm{H}+\mathrm{C}$ : gallinaza + paja + huevo + ceniza

Los únicos tratamientos que no presentan diferencias significativas son el testigo $(\mathrm{G}+\mathrm{P})$ con el tratamiento $\mathrm{G}+\mathrm{P}+\mathrm{H}+\mathrm{C}$, lo cual puede deberse a que la conductividad quede afectada por la interacción del huevo con la ceniza (Cuadro III)

CUADRO III. PRUEBA DE DIFERENCIA MÍNIMA SIGNIFICATIVA (LSD, POR SUS SIGLAS EN INGLÉS) PARA LA CONDUCTIVIDAD ELÉCTRICA (CE dS/m) POR TRATAMIENTO (MÉTODO $95.0 \%$ LSD)

\begin{tabular}{lccc}
\hline Tratamientos & Repeticiones & Medias & $\begin{array}{c}\text { Grupos } \\
\text { homogéneos }\end{array}$ \\
\hline Testigo & 3 & 5.23 & $\mathrm{C}$ \\
$\mathrm{G}+\mathrm{P}+\mathrm{C}$ & 3 & 4.13 & $\mathrm{~B}$ \\
$\mathrm{G}+\mathrm{P}+\mathrm{H}$ & 3 & 3.40 & $\mathrm{~A}$ \\
$\mathrm{G}+\mathrm{P}+\mathrm{H}+\mathrm{C}$ & 3 & 4.91 & $\mathrm{C}$ \\
\hline
\end{tabular}

Valores seguidos de la misma letra en la misma columna no difieren significativamente según la prueba LSD $(\mathrm{p}<0.05)$. Testigo: gallinaza + paja $(\mathrm{G}+\mathrm{P}) ; \mathrm{G}+\mathrm{P}+\mathrm{C}$ : gallinaza + paja + ceniza; $\mathrm{G}+\mathrm{P}+\mathrm{H}$ : gallinaza + paja + huevo; $\mathrm{G}+\mathrm{P}+\mathrm{H}+\mathrm{C}$ : gallinaza + paja + huevo + ceniza

En el caso de la pila (Fig. 8), la $\mathrm{CE}$ al inicio del proceso presentó un valor de $6.24 \pm 1.35 \mathrm{dS} / \mathrm{m}$ y a partir de la segunda semana se observó una disminución acelerada debido a la lixiviación de las sales durante el proceso (Leconte et al. 2009), para posteriormente estabilizarse a la sexta semana. Al final del proceso la CE fue de $3.86 \pm 0.84 \mathrm{dS} / \mathrm{m}$, valor aceptable según el RD 506/2013 (BOE 2013) para las enmiendas de suelos y abonos orgánicos. 


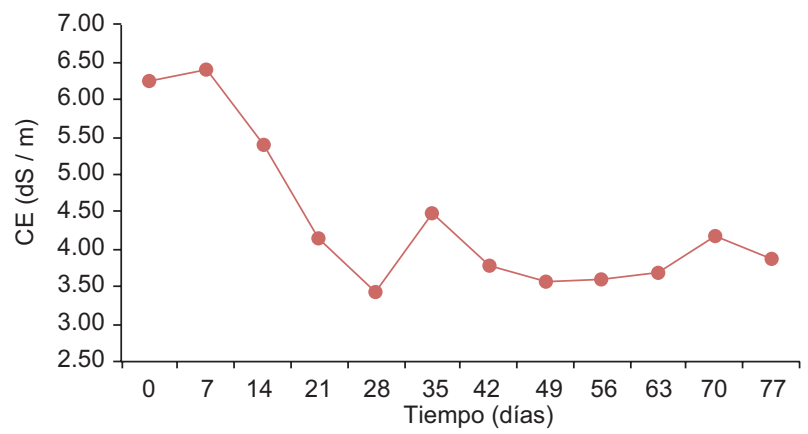

Fig. 8. Variación de la conductividad eléctrica (CE) con el tiempo del tratamiento gallinaza + paja en la pila

\section{Materia orgánica (MO)}

En el estudio sobre la evolución de la MO en los diferentes tratamientos se observó una disminución debida a la mineralización y pérdida de carbono en forma de anhídrido carbónico (Fig. 9). Este porcentaje fue disminuyendo debido a la mineralización; por consiguiente ocurrió pérdida de carbono en forma de anhídrido carbónico que llegó a representar casi el $20 \%$ en peso de la masa compostada de acuerdo con Zucconi et al. (1987). La disminución de materia orgánica ocurre en dos etapas: en la primera hay disminución de carbohidratos, que se transforman en cadenas carbonadas largas y cortas, las cuales se reagrupan para compuestos húmicos. En la segunda etapa, los materiales más resistentes como las ligninas se van degradando y/o transformando en compuestos húmicos (Tomati et al. 2000, Castaldi et al. 2005).

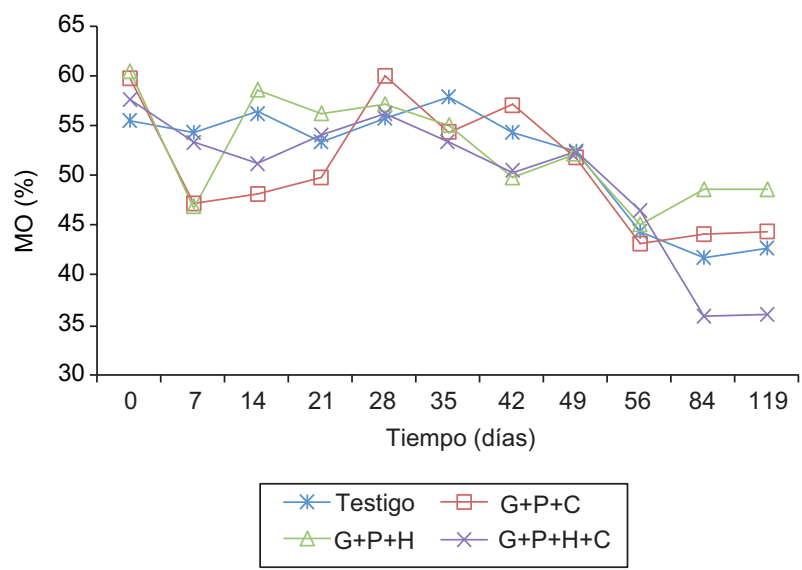

Fig. 9. Variación de la materia orgánica (MO) con el tiempo de los distintos tratamientos testigo en el ensayo a pequeña escala: gallinaza + paja $(\mathrm{G}+\mathrm{P}) ; \mathrm{G}+\mathrm{P}+\mathrm{C}$ : gallinaza + paja + ceniza; $\mathrm{G}+\mathrm{P}+\mathrm{H}$ : gallinaza + paja + huevo; $\mathrm{G}+$ $\mathrm{P}+\mathrm{H}+\mathrm{C}$ : gallinaza + paja + huevo + ceniza
Al final del proceso de compostaje, la cantidad de materia orgánica en los tratamientos testigo $(\mathrm{G}+\mathrm{P})$, $\mathrm{G}+\mathrm{P}+\mathrm{C}$ y G $+\mathrm{P}+\mathrm{H}$, se mantuvieron sin variación en $42.70 \pm 4.9 \%, 44.26 \pm 5.00 \%$ y $48.64 \pm 5.58 \%$, respectivamente, siendo el tratamiento $\mathrm{G}+\mathrm{P}+\mathrm{H}+\mathrm{C}$ el que presentó el valor más bajo $(36.11 \pm 4.14 \%)$ de MO. Todos los tratamientos superaron el contenido de $\mathrm{MO}$ del $25 \%$, por lo que el proceso fue adecuado y los resultados fueron los esperados. Los únicos tratamientos que no presentaron diferencias significativas fueron el testigo $(\mathrm{G}+\mathrm{P})$ con el tratamiento $\mathrm{G}+\mathrm{P}+\mathrm{H}$ (Cuadro IV).

CUADRO IV. PRUEBA DE DIFERENCIA MÍNIMA SIGNIFICATIVA (LSD, POR SUS SIGLAS EN INGLÉS) PARA LA MATERIA ORGÁNICA (\% MO) POR TRATAMIENTO (MÉTODO $95.0 \%$ LSD)

\begin{tabular}{lccc}
\hline Tratamientos & Repeticiones & Medias & $\begin{array}{c}\text { Grupos } \\
\text { homogéneos }\end{array}$ \\
\hline Testigo & 3 & 42.70 & B \\
G + P + C & 3 & 44.26 & C \\
G + P + H & 3 & 48.65 & B \\
G + P + H + C & 3 & 36.12 & A \\
\hline
\end{tabular}

Valores seguidos de la misma letra en la misma columna no difieren significativamente según la prueba LSD $(p<0.05)$. Testigo: gallinaza + paja $(\mathrm{G}+\mathrm{P}) ; \mathrm{G}+\mathrm{P}+\mathrm{C}$ : gallinaza + paja + ceniza; $\mathrm{G}+\mathrm{P}+\mathrm{H}$ : gallinaza + paja + huevo; $\mathrm{G}+\mathrm{P}+\mathrm{H}+\mathrm{C}$ : gallinaza + paja + huevo + ceniza

El porcentaje de MO inicial en la pila (Fig. 10) fue de $47.82 \pm 5.49 \%$, el cual se redujo durante la etapa termófila (día 28). Esto puede atribuirse a una mayor actividad microbiana y por lo tanto a una descomposición más rápida de la materia orgánica. La mayor proporción de carbono se encuentra en la

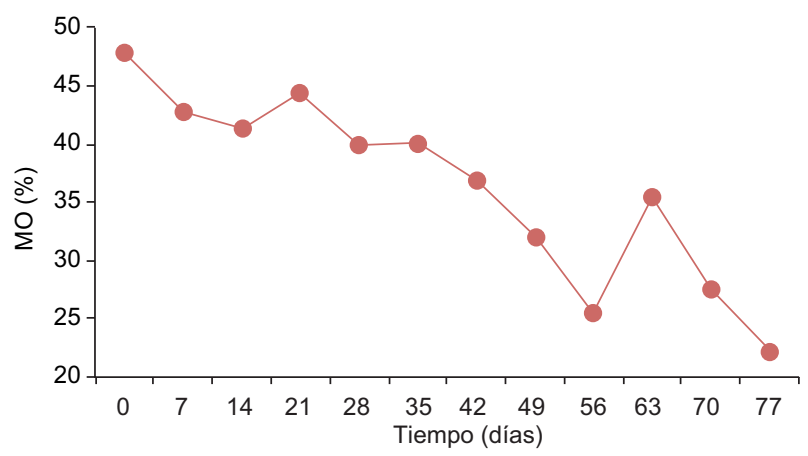

Fig. 10. Variación de materia orgánica $(\mathrm{MO})$ con el tiempo del tratamiento gallinaza + paja $(\mathrm{G}+\mathrm{P})$ en la pila 
primera etapa del compostaje; a medida que avanza el proceso de compostaje, este carbono es consumido y se transforma en humus. A los 63 días se observó incremento de la materia orgánica, quizás debido a un aumento en la temperatura en el proceso de compostaje, para estabilizarse posteriormente, a los 77 días, con una degradación de la materia orgánica de $22.32 \pm 1.20 \%$, lo cual es indicativo de la estabilidad del compost (Bernal et al. 1998).

\section{Nitrógeno}

En la figura 11 se representa el comportamiento del nitrógeno en el proceso de compostaje, siendo mayor su porcentaje en el control (3.55\%), seguido del tratamiento $\mathrm{G}+\mathrm{P}+\mathrm{H}(3.05 \pm 0.73 \%)$ y el tratamiento $\mathrm{G}+\mathrm{P}+\mathrm{H}+\mathrm{C}(2.31 \pm 0.56 \%)$. El porcentaje de nitrógeno disminuyó significativamente a los 84 días, debido a la pérdida de este elemento en forma amoniacal $\left(\mathrm{NH}_{3}\right)$. Los porcentajes de nitrógeno se encuentran entre 2.0 y $3.55 \%$ (Soto 2003), superando el $1 \%$ del contenido de nitrógeno recogido en el RD 506/2013 (BOE 2013).

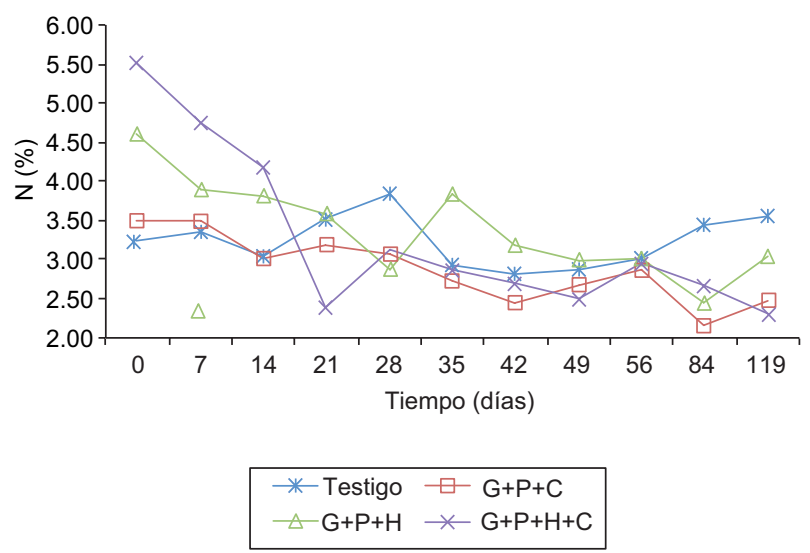

Fig. 11. Variación del nitrógeno total $(\mathrm{N})$ con el tiempo de los distintos tratamientos testigo en el ensayo a pequeña escala: gallinaza + paja $(\mathrm{G}+\mathrm{P}) ; \mathrm{G}+\mathrm{P}+\mathrm{C}$ : gallinaza + paja + ceniza; $\mathrm{G}+\mathrm{P}+\mathrm{H}$ : gallinaza + paja + huevo; $\mathrm{G}+$ $\mathrm{P}+\mathrm{H}+\mathrm{C}$ : gallinaza + paja + huevo + ceniza

Hansen et al. (1989) reportaron una disminución del $33 \%$ de nitrógeno durante el proceso de compostaje de estiércol de ave de corral y también encontraron que la volatilización del nitrógeno de residuos avícolas aumenta cuando los valores de $\mathrm{pH}$ superan a 7. En este estudio los valores de $\mathrm{pH}$ en la semana ocho se encontraban por encima de 7, lo cual podría explicar las pérdidas de nitrógeno, mediante la volatilización, como amoníaco.
Todos los tratamientos presentan diferencias estadísticamente significativas $(\mathrm{p}<0.05)(\mathbf{C u a d r o} \mathbf{V})$.

Respecto al nitrógeno en la pila (Fig. 12), entre los días 21 y 49 se observó una disminución, debido a la volatilización de este elemento (Koenig et al. 2005), proceso que se incrementó cuando los valores de $\mathrm{pH}$ fueron mayores de 7.00. Esto explicaría la pérdida de nitrógeno por volatilización como amoniaco (Groot y Elzing 1996).

CUADRO V. PRUEBA DE DIFERENCIA MÍNIMA SIGNIFICATIVA(LSD, POR SUS SIGLAS EN INGLÉS) PARA NITRÓGENO $(\%$ N) POR TRATAMIENTO (MÉTODO $95.0 \%$ LSD)

\begin{tabular}{lccc}
\hline Tratamientos & Repeticiones & Medias & $\begin{array}{c}\text { Grupos } \\
\text { homogéneos }\end{array}$ \\
\hline Testigo & 3 & 3.55 & $\mathrm{D}$ \\
$\mathrm{G}+\mathrm{P}+\mathrm{C}$ & 3 & 2.47 & $\mathrm{~B}$ \\
$\mathrm{G}+\mathrm{P}+\mathrm{H}$ & 3 & 3.05 & $\mathrm{C}$ \\
$\mathrm{G}+\mathrm{P}+\mathrm{H}+\mathrm{C}$ & 3 & 2.31 & $\mathrm{~A}$ \\
\hline
\end{tabular}

Valores seguidos de la misma letra en la misma columna no difieren significativamente según la prueba LSD $(p<0.05)$. Testigo: gallinaza + paja $(\mathrm{G}+\mathrm{P}) ; \mathrm{G}+\mathrm{P}+\mathrm{C}$ : gallinaza + paja + ceniza; $\mathrm{G}+\mathrm{P}+\mathrm{H}$ : gallinaza + paja + huevo; $\mathrm{G}+\mathrm{P}+\mathrm{H}+\mathrm{C}$ : gallinaza + paja + huevo + ceniza

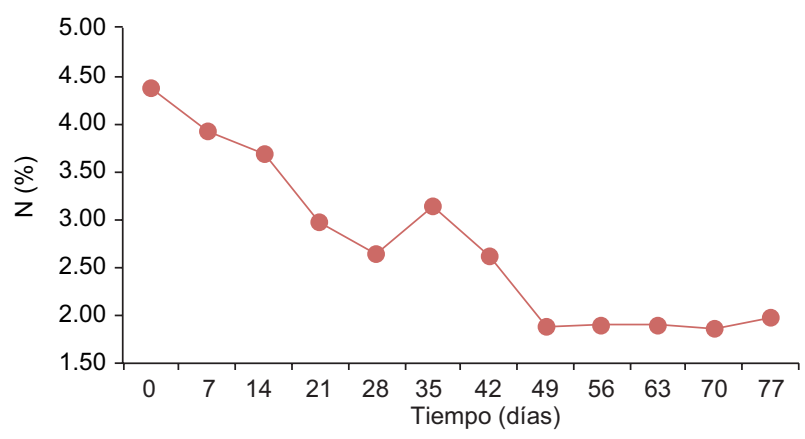

Fig. 12. Variación de nitrógeno total (N) con el tiempo del tratamiento gallinaza + paja en la pila

\section{Relación C/N}

Para un correcto compostaje, la relación $\mathrm{C} / \mathrm{N}$ debe ser la adecuada, ya que es un factor importante en la evolución del proceso y la pérdida de amonio (Jhorar et al. 1991). Los resultados de la relación $\mathrm{C} / \mathrm{N}$ se muestran en la figura 13, donde se observa que el control y los tratamientos tuvieron una relación $\mathrm{C} / \mathrm{N}<20$. El testigo $(\mathrm{G}+\mathrm{P})$ presentó el menor valor $(12.03 \pm 0.17)$, lo cual se atribuye a una mayor cantidad de microorganismos que actúan sobre la 
MO consumiendo el carbono presente en la mezcla. El tratamiento $\mathrm{G}+\mathrm{P}+\mathrm{C}$ presentó el valor más alto $(17.92 \pm 0.25)$ debido a que los microorganismos no alcanzaron a consumir todo el carbono orgánico aportado al inicio del compostaje.

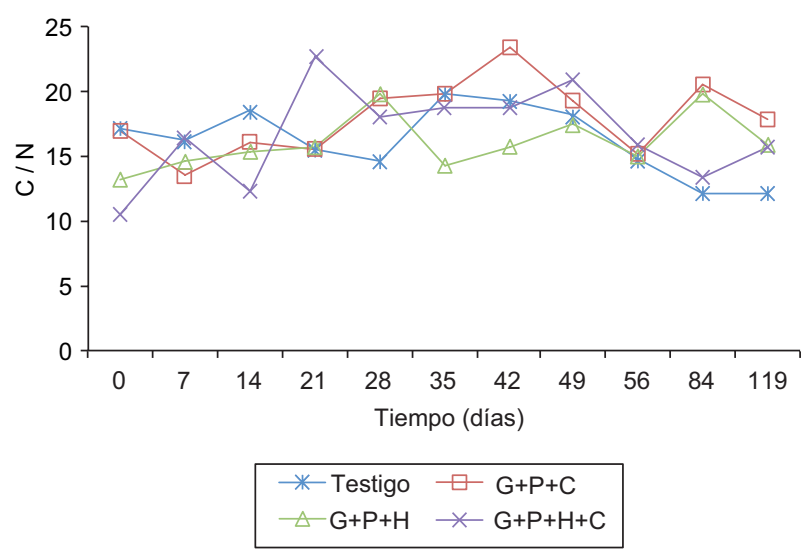

Fig. 13. Variación de la relación carbono:nitrógeno $(\mathrm{C} / \mathrm{N})$ con el tiempo de los distintos tratamientos testigo en el ensayo a pequeña escala: gallinaza + paja $(\mathrm{G}+\mathrm{P}) ; \mathrm{G}+\mathrm{P}+\mathrm{C}$ : gallinaza + paja + ceniza; $\mathrm{G}+\mathrm{P}+\mathrm{H}$ : gallinaza + paja + huevo; $\mathrm{G}+\mathrm{P}+\mathrm{H}+\mathrm{C}$ : gallinaza + paja + huevo + ceniza

En el caso de la pila, la relación $\mathrm{C} / \mathrm{N}$ al iniciar el proceso presentó un valor de $10.74 \pm 0.15$ (Fig. 14), siendo el carbono superior al nitrógeno, ya que los microrganismos lo utilizan como fuente de energía y el nitrógeno para la formación de la biomasa microbiana (Khan et al. 2014). Por tal motivo la relación $\mathrm{C} / \mathrm{N}$ debe ser equilibrada, ya que es un indicador que sirve para evaluar el desarrollo y calidad de compostaje (Guo et al. 2012). Al final del proceso la relación $\mathrm{C} / \mathrm{N}$ presentó un valor de $11.27 \pm 0.15$ debido a que gran parte del carbono es

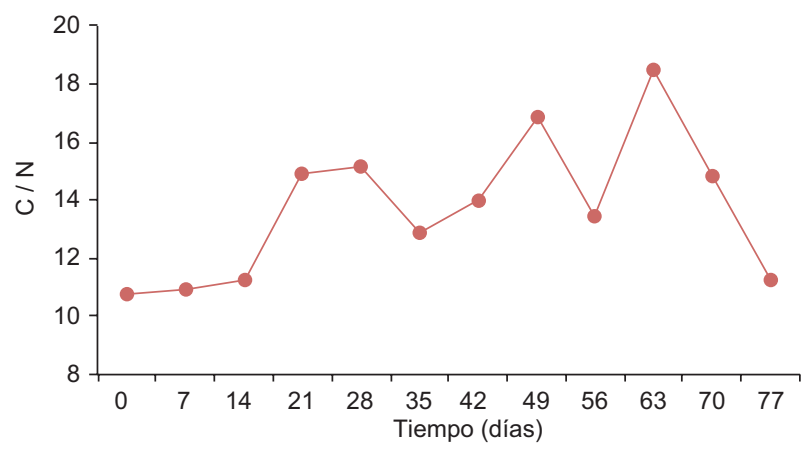

Fig. 14. Variación de la relación carbono:nitrógeno $\mathrm{C} / \mathrm{N}$ con el tiempo del tratamiento gallinaza + paja en la pila liberado $\left(\mathrm{CO}_{2}\right)$ y la mayoría del nitrógeno reciclado, lo que indica la descomposición de la materia orgánica y su estabilización. Si la relación $\mathrm{C} / \mathrm{N}$ es mayor de 40 la actividad microbiana disminuye y los microorganismos oxidan el carbono, provocando que el tiempo de proceso de compost se prolongue debido a la baja disponibilidad de nitrógeno. Según los datos obtenidos en los dos ensayos tanto a pequeña escala como a escala semiindustrial respecto de la relación $\mathrm{C} / \mathrm{N}<20$, los compost resultantes han alcanzado una gran estabilidad y madurez.

$\mathrm{Al}$ tener la relación $\mathrm{C} / \mathrm{N}$ una gran dependencia del nitrógeno, éste no refleja el estado de madurez del compost, por lo que es recomendable seguir la evolución de la relación $\mathrm{C} / \mathrm{N}$ en todo el proceso y calcular la diferencia entre los valores iniciales y finales (Guo et al. 2012).

\section{Fósforo}

El fósforo desempeña un papel muy importante en la formación de compuestos celulares ricos en energía, indispensables para el metabolismo microbiano. Es el nutriente más importante después del carbono y el nitrógeno, encontrándose en el suelo en concentraciones de 200 a $5000 \mathrm{mg} / \mathrm{kg}$.

Sólo se realizaron análisis al inicio y al final del compostaje para el ensayo a pequeña escala. Las concentraciones al inicio del compostaje fueron de $3291,2819,3352$ y $2896 \mathrm{mg} / \mathrm{kg}$ para los tratamientos testigo $(\mathrm{G}+\mathrm{P}), \mathrm{G}+\mathrm{P}+\mathrm{C}, \mathrm{G}+\mathrm{P}+\mathrm{H}$ y G $+\mathrm{P}+\mathrm{H}+$ $\mathrm{C}$, respectivamente.

Todos los tratamientos presentan diferencias estadísticamente significativas $(\mathrm{p}<0.05)$ excepto el tratamiento $\mathrm{G}+\mathrm{P}+\mathrm{C}$ con el tratamiento $\mathrm{G}+\mathrm{P}+\mathrm{H}$ $+\mathrm{C}$ (Cuadro VI).

CUADRO VI. PRUEBA DE DIFERENCIA MÍNIMA SIGNIFICATIVA (LSD, POR SUS SIGLAS EN INGLÉS) PARA EL FÓSFORO (P [mg/kg]) POR TRATAMIENTO (MÉTODO 95.0\% LSD)

\begin{tabular}{lccc}
\hline Tratamientos & Repeticiones & Medias & $\begin{array}{c}\text { Grupos } \\
\text { homogéneos }\end{array}$ \\
\hline Testigo & 3 & 4374.00 & $\mathrm{C}$ \\
$\mathrm{G}+\mathrm{P}+\mathrm{C}$ & 3 & 2819.00 & $\mathrm{~B}$ \\
$\mathrm{G}+\mathrm{P}+\mathrm{H}$ & 3 & 2613.00 & $\mathrm{~A}$ \\
$\mathrm{G}+\mathrm{P}+\mathrm{H}+\mathrm{C}$ & 3 & 2896.00 & $\mathrm{~B}$ \\
\hline
\end{tabular}

Valores seguidos de la misma letra en la misma columna no difieren significativamente según la prueba LSD $(p<0.05)$. Testigo: gallinaza + paja $(\mathrm{G}+\mathrm{P}) ; \mathrm{G}+\mathrm{P}+\mathrm{C}$ : gallinaza + paja + ceniza; $\mathrm{G}+\mathrm{P}+\mathrm{H}$ : gallinaza + paja + huevo; $\mathrm{G}+\mathrm{P}+\mathrm{H}+\mathrm{C}$ : gallinaza + paja + huevo + ceniza 
Respecto al contenido de fósforo en el ensayo en la pila (Fig. 15), se puede comprobar la misma tendencia que en el ensayo a pequeña escala con un aumento de este elemento del inicio al final del ensayo con la obtención de la composta de acuerdo con Wei et al. (2015).

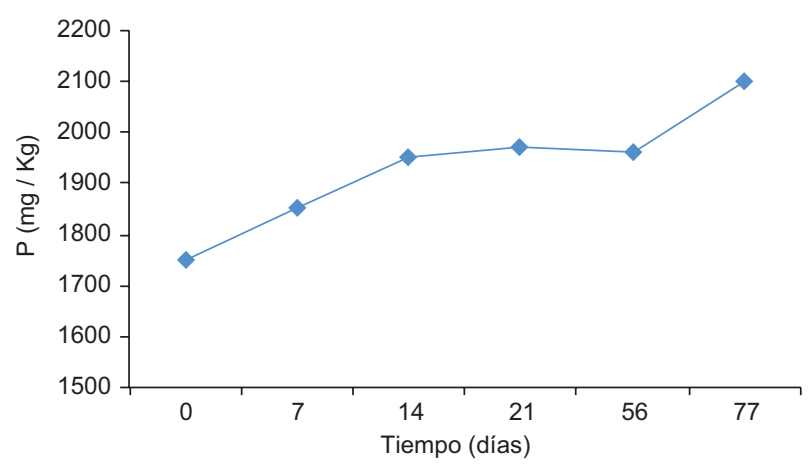

Fig. 15. Variación del fósforo $(\mathrm{mg} / \mathrm{kg})$ con el tiempo del tratamiento gallinaza + paja en la pila

\section{Bioensayo de fitotoxicidad}

Para realizar este bioensayo los resultados de los tratamientos fueron comparados con un compost comercial. En la figura 16 se muestran los resultados obtenidos del bioensayo de fitoxicidad, con un índice de germinación del $100 \%$ para el compost comercial y el control (ensayo con agua). En el caso de los tratamientos $(\mathrm{G}+\mathrm{P}+\mathrm{H}, \mathrm{G}+\mathrm{P}+\mathrm{C}$ y $\mathrm{G}+\mathrm{P}$ $+\mathrm{H}+\mathrm{C}$ ) el índice de germinación fue del 65, $62 \mathrm{y}$ $68 \%$, respectivamente, y para el control $(\mathrm{G}+\mathrm{P})$, del $75 \%$. Por último, en la pila se obtuvo un índice

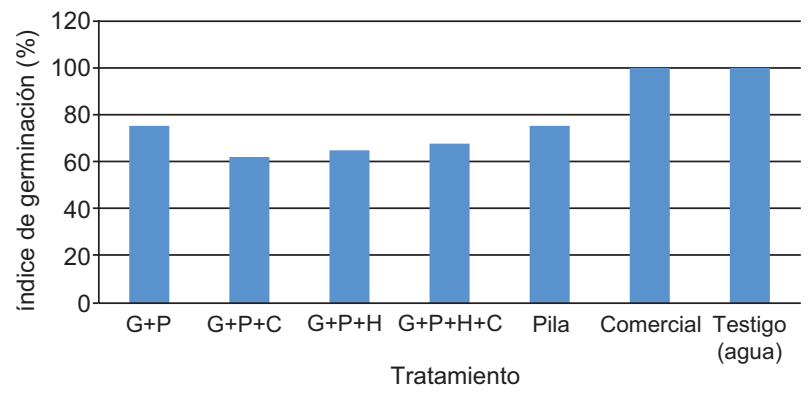

Fig. 16. Índice de germinación de los distintos tratamientos testigo en el ensayo a pequeña escala $(\mathrm{G}+\mathrm{P}$ : gallinaza + paja; G + P + C: gallinaza + paja + ceniza; $\mathrm{G}+\mathrm{P}+$ $\mathrm{H}$ : gallinaza + paja + huevo; $\mathrm{G}+\mathrm{P}+\mathrm{H}+\mathrm{C}$ : gallinaza + paja + huevo + ceniza), en la pila $(\mathrm{G}+\mathrm{P}$ : gallinaza + paja), en el compost comercial y en el testigo (con agua) de germinación del $78 \%$. Con estos índices de toxicidad se puede observar la madurez del compost de cada tratamiento. Por tanto, los compost elaborados con $\mathrm{G}+\mathrm{P}$, tanto a pequeña escala como a escala semiindustrial, se comportaron de manera similar y ambos pueden poponerse para uso agrícola. No hubo diferencias significativas entre tratamientos $(G+P$, $\mathrm{G}+\mathrm{P}+\mathrm{C}, \mathrm{G}+\mathrm{P}+\mathrm{H}$ y G $+\mathrm{P}+\mathrm{H}+\mathrm{C}$ ) en el ensayo a pequeña escala $(p<0.05)$, pero sí se encontraron diferencias significativas entre el control (agua) y el compost comercial (Fig. 16).

Los análisis microbiológicos del compost tanto en el ensayo a pequeña escala como a escala semiindustrial mostraron todos los tratamientos cumplen con la normativa propuesta en el RD 506/2013 (BOE 2013) de los compuestos orgánicos usados como abonos o fertilizantes. Salmonella estuvo ausente en $25 \mathrm{~g}$ y Escherichia coli $<1000 \mathrm{NMP} / \mathrm{g}$.

\section{CONCLUSIONES}

Al finalizar el proceso de compostaje de residuos avícolas, la relación $\mathrm{C} / \mathrm{N}$ fue $<20$ en los ensayos realizados con diferentes sustratos, tanto a nivel de pequeña escala como a escala semiindustrial. Dicha relación indica un compost estable y de buena calidad que cumple con el RD 506/2013 (BOE 2013) de los compuestos orgánicos usados como abonos o fertilizantes.

Asimismo, al finalizar el proceso compostaje los parámetros estudiados, como temperatura, $\mathrm{pH}$, conductividad eléctrica, materia orgánica y humedad, se encontraron dentro de los niveles óptimos requeridos para abonos orgánicos constituidos, total o parcialmente, por residuos orgánicos biodegradables.

Los parámetros finales encontrados en una pila de compostaje semiindustrial en condiciones ambientales fueron los adecuados para un compost maduro.

Desde el punto de vista fitotóxico se pudo determinar que ninguno de los tratamientos ensayados dio lugar a un compost tóxico para las plantas, y con relación a su análisis microbiológico se comprobó que todos los tratamientos cumplen con la normativa, por lo que pueden utilizarse para usos agrícolas o forestales, o bien como mejoradores de suelo.

\section{AGRADECIMIENTOS}

Este estudio se realizó gracias al proyecto RTA201400012-CO3-01 (INIA-FEDER). Los autores 
agradecen a Jesús García, Beatriz Albero, Esther de Miguel y Ángela García su colaboración en las tareas de campo y laboratorio.

\section{REFERENCIAS}

Arrigo N., Jiménez M.P., Palma R. y Tortarolo M.F. (2005). Residuos de poda compostados sin compostar: uso potencial como enmienda orgánica en suelo. Suelo 23 (1), 87-92.

Alexander M. (1977). Introduction to soil microbiology. 2a ed. John Wiley and Sons, Nueva York, EUA, 467 pp.

APHA-AWWA-WPCF (2005). Standard methods for the examination of water and wastewater. 21a ed. American Public Health Association-American Water Works Assotiation-Water Environment Federation, Washington, EUA, 874 pp.

Bernal M.P., Paredes C., Sánchez-Monedero M.A. y Cegarra J. (1998). Maturity and stability parameters of composts prepared with a wide range of organic wastes. Bioresour. Technol. 63 (1), 91-99.

DOI: $10.1016 / \mathrm{s} 0960-8524(97) 00084-9$

BOE 2013. Real Decreto 506/2013. Sobre productos fertilizantes. Boletín Oficial del Estado, 10 de julio [en línea]. https://www.boe.es/eli/es/rd/2013/06/28/506

Castaldi P., Alberti G., Merella R. y Melis P. (2005). Study of the organic matter evolution during municipal solid waste composting aimed at identifying suitable parameters for the evaluation of compost maturity. Waste Manag. 25 (2), 209-213.

DOI: $10.1016 /$ j.wasman.2004.12.011

Celaya H. y Castellanos A. (2011). Mineralización de nitrógeno en el suelo de zonas áridas y semiáridas. Terra Latinoamericana 29, 343-356.

DOI: $10.1007 /$ s00374-009-0389-4

Delgado M., Miralles de Imperial R., Martín J., León C. y García M. (2007). Evaluación de residuos orgánicos de origen animal procedentes de granjas avícolas. Revista Ingeniería de Recursos Naturales y del Ambiente 6, 33-39.

Delgado M., Martín J.V., Miralles de Imperial R., León C. y García M.C. (2010). Phytotoxicity of uncomposted and composted poultry manure. AJP Academic Journal 4 (5), 154-162.

Escudero de Fonseca A. y Arias C. (2012). Los microorganismos en abonos orgánicos a partir de podas en la Universidad del Norte, Colombia. Rev. Int. Contam. Ambie. 28 (Sup. 1), 67-75.

Fang M. y Wong J. (1999). Co-composting of sewage sludge and coal fly ash: Nutrient transformation. Bioresour. Technol. 67 (1), 19-24.

DOI: $10.1016 /$ S0960-8524(99)00095-4
Groot Koerkamp P.W.G. y Elzing A. (1996). Degradation of nitrogenous components in and volatilization of $\mathrm{NH}_{3}$ from litter in aviary housing systems for laying hens. Transactions ASAE 39, 211-218.

Guenon R. y Gros R. (2015). Increasing the maturity of compost used affects the soil chemical properties and the stability of microbial activity along a Mediterranean post-fire chronosequence. Eur. J. Soil Biol. $66,1-10$

DOI: 10.1016/j.ejsobi.2014.11.002

Guerra E., Vásquez M. y Díaz M. (2001). Dynamics of physicochemical and biological parameters during the co-composting of chestnut burr/leaf litter with solid poultry manure. J. Sci. Food and Agri. 81, 648-652. DOI: 101002/jsfa-866/abstract

Guo R., Li G. y Jiang T. (2012). Effect of aeration rate, $\mathrm{C} / \mathrm{N}$ ratio and moisture content on the stability and maturity of compost. Bioresour. Technol. 112, 171-178. DOI: 10.101016/j.biotech 2012.02.099

Hansen R.C, Keener H.M. y Hoitink H.A. (1989). Poultry manure composting. An exploratory study. T. ASAE 36, 2151-2157. DOI:10.13031/2013.31277

Haug R.T. (1993). The practical handbook of compost engineering. Lewis Publishers, Boca Ratón, EUA, $388 \mathrm{pp}$.

Hernández S. y Rodríguez O. (2013). Calidad nutrimental de cuatro abonos orgánicos producidos a partir de residuos vegetales y pecuarios. Terra Latinoam. 31 (1), 35-46.

Hesse P.R. (1971). Total nitrogen. The Kjeldahl process. En: A textbook of soil chemical analysis. Murray, Gran Bretaña, 520 pp.

Jhorar B.S., Phogat V. y Malik E. (1991). Kinetics of composting rice straw with glue waste at different carbon:nitrogen ratios in a semiarid environment. Arid Soil Rest. Rehabil. 5, 297-306.

Khan N., Clark L., Sánchez M.M., Shea S., Meier S. y Bolan N. (2014). Maturity indices in co-composting of chicken manure and saw dust with biochar. Bioresour. Technol. 168, 245-251.

DOI: 10.1016/j.biotech.2014.02.123

Koenig R.T., Dean F., Miller B.E. y Harrison J.D. (2005) Variability of atmospheric ammonia in high-rise, caged layer composting. Compost Sci. Util. 13 (3), 162-167. DOI: $10.1080 / 1056657 X .20115 .10702236$

Leconte M.C., Mazzarino M.J., Satti P., Iglesias M.C. y Laos F. (2009). Composting poultry manure with rice hulls and/or sawdust in NE Argentina. Waste Manage. 29, 2446-2453. DOI: 10.101016/1.wasman.2009.006

Liao Q., Huang D. y Jiang Z. (2013). Estimation of production amount of livestock and poultry manure and environmental impact assessment in Guangxi. Asian Agric. Res. 5, 93-96. 
Magdi T., Takatsugo H. y Shinga O. (2004). Composting of rice straw with oilseed rape cake and poultry manure and its effects on faba bean (Vicia faba L.) growth and soil properties. Biores. Technol. 93, 183-189.

DOI: $10.1016 /$ j.biortech.2003.10.012

MAGRAMA (2014). Base de datos. Ministerio de Agricultura, Alimentación y Medio Ambiente de España [en línea]. http://www.magrama.gob.es/ganaderia/temas/ sanidad 25/02/16

MAPA (1994). Métodos oficiales de análisis. Ministerio de Agricultura, Pesca y Alimentación. Madrid, España, $662 \mathrm{pp}$.

Matheus L., Graterol B., Simancas G. y Fernández O. (2007). Eficiencia agronómica relativa de tres abonos orgánicos (vermicompost, compost, y gallinaza) en plantas de maíz (Zea mays L). Agricultura Andina 13, 27-38.

Mathur P. (1991). Composting processes. En: Bioconversion of waste materials to industrial products (A.M. Martin, Ed.). Elsevier, Londres, Gran Bretaña, pp. 147-183.

Morisaki N., Nakasaki K., Shoda M. y Kubota H. (1989). Nitrogen transformation during thermophilic composting. J. Ferment. Bioeng. 67 (1), 57-61.

DOI: $10.1016 / 0922-338 X(89) 90087-1$

Nieto A. (2002). El uso de compostas como alternativa ecológica para la producción sostenible del chile (Capsicum annuum L.) en zonas áridas. Interciencia 27 (8), 417- 421.

Pino G.P., Varnero M.T. y Alvarado V.P. (2005). Dinámica del compostaje de residuos vitivinícolas con y sin incorporación de guano Broiler. Rev. Cienc. Suelo Nutr. 5 (2), 19-25.

Ramírez F., Fournier M.L., Ruepert C. e Hidalgo C. (2014). Uso de agroquímicos en el cultivo de papa en Pacayas, Cartago, Costa Rica. Agron. Mesoam. 25 (2), 337-345.

Roca L., Martínez P. y Marcilla R. (2008). Composting rice straw with sewage sludge and compost effects on the soil-plant system. Chesmosphere 75 (6), 781-787. DOI: 10.1016/j. chesmosphere 200812058

Riera N.I. Della T.V., Rizzo P.F., Bultti M., Bressan F.M., Zarate N. y Crespo D.E. (2014). Evaluación del proceso de compostaje de dos mezclas de residuos avícolas. Rev. Fac. Cienc. Agrar. 46 (1), 195-203.
Sánchez-Monedero M.A., Roig A., Paredes C. y Bernal M. (2001). Nitrogen transformation during organic waste composting by the Rutgers system and its effects on $\mathrm{pH}, \mathrm{EC}$ and maturity of the composting mixtures. Biores. Technol. 78, 301-308. DOI: 10.1016/S0960-8524(01)00031-1

SAS (2004). SAS/STAT user's guide. Virgina Clark, Carolina del Norte, EUA, 4975 pp.

Smith K.A., Brewer A.J., Crabb J y Dauven A. (2001). A survey of the production and use of animal manures in England and Wales. Poultry manure. Soil Use Manage. 5, 17-48. DOI: 10.1111/j.1475-2743.2001.tb00012.X

Soto M.G. (2003). Abonos orgánicos: definiciones y procesos. En: Abonos orgánicos: principios, aplicaciones e impactos en la agricultura. (G. Meléndez, Ed.). EUNED, San José, Costa Rica, pp. 20-49.

Sztern D. y Pravia M. (2008). Manual para la elaboración de compost. Bases conceptuales y procedimientos. Tacuarembó, Uruguay, 69 pp.

Tautges N., Borrelli K., Burke I. y Fuerst P. (2018). Nitrogen fertility effects of alfalfa, pea green manure and poultry manure on organic wheat productivity in a semiarid climate. Agroecology Sustain. Food Syst. 42 (2), 169-188. DOI: 10.1080/21683565.20171380739

Tomati U., Madejon E. y Galli E. (2000). Evolution of humic acid molecular weight as an index of compost stability. Compost Sci. Util. 8 (2), 108-115.

DOI: $10.1080 / 1065657 X .2000 .10701756$

Torrentó M. (2011). Materia orgánica y compostaje. Control de la calidad y del proceso. Jornada Técnica: Fertilidad y Calidad del Suelo. Instituto Canario de Investigaciones Agrarias, Santiago, Chile, 19 pp.

Wei Y., Zhao Y., Xi B., Wei Z., Li X. y Cao Z. (2015). Changes in phosphorus fractions during organic wastes composting from different sources. Biores. Technol. 189, 349-356. DOI: 10.1016/j. biotech 2015.04.031

Zucconi F. y Bertoldi M. (1987). Compost specifications for the production and characterization of compost from municipal solid waste. Applied Sci. 28, 30-50. 